\title{
Dynamics of Distributed Updating in Fisher Markets
}

\author{
YUN KUEN CHEUNG, Max Planck Institute for Informatics, Saarland Informatics Campus, Germany \\ RICHARD COLE and YIXIN TAO, Courant Institute, NYU, USA
}

A major goal in Algorithmic Game Theory is to justify equilibrium concepts from an algorithmic and complexity
perspective. One appealing approach is to identify natural distributed algorithms that converge quickly to an
equilibrium. This paper established new convergence results for two generalizations of proportional response
in Fisher markets with buyers having CES utility functions. The starting points are respectively a new convex
and a new convex-concave formulation of such markets. The two generalizations correspond to suitable
mirror descent algorithms applied to these formulations. Several of our new results are a consequence of new
notions of strong Bregman convexity and of strong Bregman convex-concave functions, and associated linear
rates of convergence, which may be of independent interest.

Among other results, we analyze a damped generalized proportional response and show a linear rate of convergence in a Fisher market with buyers whose utility functions cover the full spectrum of CES utilities aside the extremes of linear and Leontief utilities; when these utilities are included, we obtain an empirical $O(1 / T)$ rate of convergence.

CCS Concepts: • Theory of computation $\rightarrow$ Algorithmic game theory and mechanism design; Market equilibria;

Additional Key Words and Phrases: Proportional response, mirror descent, Bregman divergence, Fisher market.

\section{INTRODUCTION}

One of the most important results in Algorithmic Game Theory is the PPAD-hardness of finding a Nash Equilibrium in finite games [8,17], which serves as a strong evidence that there is no efficient algorithm to compute Nash Equilibria. Similar hardness results have been established for markets $[7,9,10,14,47]$. By viewing the players and the environment collectively as implicitly performing a computation, these hardness results indicate that, in general, a market cannot reach an equilibrium quickly. In Kamal Jain's words: "If your laptop cannot find it, neither can the market" [40, Chapter 2.1].

As a result, a lot of attention has been given to the design of polynomial-time algorithms to find equilibria, either exactly or approximately, for specific families of games and markets. Most of these algorithms can be categorized as either simplex-like (e.g., Lemke-Howson), numerical

The work of Richard Cole and Yixin Tao was supported in part by NSF grant CCF-1527568.

Authors' addresses: Yun Kuen Cheung, Max Planck Institute for Informatics, Saarland Informatics Campus, Germany, ycheung@mpi-inf.mpg.de; Richard Cole; Yixin Tao, Courant Institute, NYU, USA, cole@cs.nyu.edu,yt851@nyu.edu.

Permission to make digital or hard copies of all or part of this work for personal or classroom use is granted without fee provided that copies are not made or distributed for profit or commercial advantage and that copies bear this notice and the full citation on the first page. Copyrights for components of this work owned by others than ACM must be honored. Abstracting with credit is permitted. To copy otherwise, or republish, to post on servers or to redistribute to lists, requires prior specific permission and/or a fee. Request permissions from permissions@acm.org.

(C) 2018 Association for Computing Machinery.

ACM EC'18, fune 18-22, 2018, Ithaca, NY, USA. ACM ISBN 978-1-4503-5829-3/18/06 ..\$15.00

https://doi.org/10.1145/3219166.3219189 
methods (e.g., the interior-point method or the ellipsoid method), or some carefully-crafted combinatorial algorithm (e.g., flow-based algorithms for computing a market equilibrium for linear utility functions).

However, it seems implausible that these algorithms describe the implicit computations in games or markets. For many markets would appear to have a highly distributed environment, or need to make rapid decisions on an ongoing basis. These features would appear to preclude computations which require centralized coordination, which is essential for the three categories of algorithms above. Consequently, in order to justify equilibrium concepts, we want natural algorithms which could plausibly be running (in an implicit form) in the associated distributed environments.

This paper will focus on Fisher markets (or economies ${ }^{1}$ ). In a Fisher market there are buyers who start with money which they have no desire to keep, and sellers who have goods to sell, which they wish to sell in their entirety for money. This is a modest generalization of the notion of Competitive Equilibrium from Equal Incomes (CEEI) [27, 46]. In prior work on computing equilibria for these markets, there has been a particular focus on Eisenberg-Gale markets, a term coined by Jain and Vazirani, and their generalizations [28]; Eisenberg-Gale markets are Fisher markets in which demands are determined by homothetic utility functions. The latter markets have been seen to capture the notion of proportional fairness, as defined in the networking community [30], which is also equivalent to the optimum Nash Social Welfare [29, 35].

Two natural dynamics have been studied in the context of Fisher markets. The first, which is perhaps the most intuitive candidate for a natural algorithm, is tatonnement, in which the price of a good is raised if the demand exceeds the supply of the good, and decreased if the demand is too small. Implicitly, buyers' demands are assumed to be a best-response to the current prices. This highly intuitive algorithm was proposed by Walras well over a century ago [48].

Proportional Response, in contrast, is a buyer-oriented update, originally analyzed in an effort to explain the behavior of peer-to-peer networks [31, 49]. Here, buyers update their spending in proportion to the contribution each good makes to its current utility. While its meaning is clear for linear and other separable utilities, for other classes of utilities this needs more interpretation, which we provide in Section 2. Here prices are assumed to equal the current spending. An $O(1 / T)$ rate of convergence was shown in [2] for Fisher markets with buyers having linear utilities, and for the substitutes domain excluding linear utilities, a faster linear rate (i.e., $\exp (-\Omega(T)$ ) rate) of convergence was shown in [50].

This paper continues the exploration of the connection between distributed dynamic processes and convex optimization, and more specifically the relation of proportional response to mirror descent.

Our first set of results starts by rederiving Zhang's bounds for CES substitutes utilities, by showing that for this setting proportional response amounts to mirror descent on a suitable convex function. To achieve the linear rate of convergence he obtained, we need to go beyond the standard $O(1 / T)$ rate of convergence for mirror descent with a Bregman divergence. We proceed by analogy with gradient descent. Gradient descent with a Lipschitz constraint on the gradients guarantees only an $O(1 / T)$ rate of convergence, but a faster linear rate of convergence is obtained when the objective function $f$ is strongly convex. For mirror descent with Bregman Divergences we introduce the notion of strong Bregman convexity and show that it also leads to a linear convergence rate. It turns out that the convex function associated with the CES substitutes utilities satisfies strong Bregman convexity, thereby obtaining Zhang's bound anew. In addition, for complementary CES

${ }^{1}$ In the CS literature the term market has been widely used to refer to economies; we follow this practice. 
utilities, the same now concave function satisfies an analogous strong Bregman concavity property, which also yields a linear rate of convergence for these utilities. In addition, if we include linear utilities in the substitutes utilities, we obtain an $O(1 / T)$ rate of convergence; likewise, including Leontief utilities in the complementary utilities also yields an $O(1 / T)$ rate.

Next, we seek to handle substitute and complementary CES utilities simultaneously. The challenge we face is that the objective function used for the first set of results becomes a mixed concave-convex function in this setting, and the equilibrium corresponds to a saddle point of this function. We introduce the further notion of strongly-Bregman convex-concave functions, and for these functions we obtain a linear rate of convergence to the saddle point. Again, our objective function of the mixed CES utilities satisfies this property, thereby yielding linear convergence, albeit now for a damped proportional response, rather than the undamped proportional response analyzed in the first set of results. Here, including linear utilities and Leontief utilities yields an empirical $O(1 / T)$ rate of convergence.

We note that our results are not a straightforward application of the existing mirror descent toolbox. The Bregman notions and the related convergence results in this paper are new. While the results for strong Bregman convex (resp. concave) functions are natural generalizations of gradient descent (resp. ascent) on standard strong convex (resp. concave) functions, the technique for demonstrating convergence for strong Bregman convex-concave functions appears to be new. It is not evident that suitable damping (i.e., reducing the step-size) permits a clean convergence analysis. Indeed, results showing linear point-wise convergence on convex-concave functions are rare ; in fact, the only such work we are aware of is [26]. We believe the new notions and convergence results for optimization problems may be of wider interest.

Roadmap. In Section 2 we give necessary definitions and notation, and in Section 3 we state our results precisely. Then, in Section 4, we describe related work. In Section 5 we carry out the analysis of mirror descent when Strong Bregman convexity holds, and then derive the convergence behavior of Proportional Response in each of the substitutes and complements domains. In Section 7, we perform an analogous analysis for strong Bregman convex-concave functions, and deduce the convergence behavior of a damped Proportional Response in combined substitutes and complements domains. Finally, in Section 9 we discuss the rates of convergence under some alternate measures.

\section{NOTATION AND DEFINITIONS}

We use bold symbols, e.g., $\mathbf{p}, \mathbf{x}, \mathbf{e}$, to denote vectors.

Fisher Market. In a Fisher market, there are $n$ perfectly divisible goods and $m$ buyers. Without loss of generality, the supply of each good $j$ is normalized to be one unit. Each buyer $i$ has a utility function $u_{i}: \mathbb{R}_{+}^{n} \rightarrow \mathbb{R}$, and a budget of size $e_{i}$. At any given price vector $\mathbf{p} \in \mathbb{R}_{+}^{n}$, each buyer purchases a maximum utility affordable collection of goods. More precisely, $\mathbf{x}_{i} \in \mathbb{R}_{+}^{n}$ is said to be a demand of buyer $i$ if $\mathbf{x}_{i} \in \arg \max _{\mathrm{x}^{\prime}: \mathbf{x}^{\prime} \cdot \mathbf{p} \leq e_{i}} u_{i}\left(\mathbf{x}^{\prime}\right)$.

A price vector $\mathbf{p}^{*} \in \mathbb{R}_{+}^{n}$ is called a market equilibrium if at $\mathbf{p}^{*}$, there exists a demand $\mathbf{x}_{i}$ of each buyer $i$ such that

$$
p_{j}^{*}>0 \Rightarrow \sum_{i=1}^{m} x_{i j}=1 \quad \text { and } \quad p_{j}^{*}=0 \Rightarrow \sum_{i=1}^{m} x_{i j} \leq 1 .
$$

The collection of $\mathbf{x}_{i}$ is said to be an equilibrium allocation to the buyers. 
CES utilities. In this paper, each buyer $i$ 's utility function is of the form

$$
u_{i}\left(\mathbf{x}_{i}\right)=\left(\sum_{j=1}^{n} a_{i j} \cdot\left(x_{i j}\right)^{\rho_{i}}\right)^{1 / \rho_{i}}
$$

for some $-\infty \leq \rho_{i} \leq 1 . u_{i}\left(\mathbf{x}_{i}\right)$ is called a Constant Elasticity of Substitution (CES) utility function. They are a class of utility functions often used in economic analysis. The limit as $\rho_{i} \rightarrow-\infty$ is called a Leontief utility, usually written as $u_{i}\left(\mathbf{x}_{i}\right)=\min _{j} \frac{x_{i j}}{c_{i j}}{ }^{2}$; and the limit as $\rho_{i} \rightarrow 0$ is called a Cobb-Douglas utility, usually written as $\prod_{j} x_{i j}{ }^{a_{i j}}$, with $\sum_{j} a_{i j}=1$. The utilities with $\rho_{i} \geq 0$ capture goods that are substitutes, and those with $\rho_{i} \leq 0$ goods that are complements.

Notation. Buyer $i$ 's spending on good $j$, denoted by $b_{i j}$, is given by $b_{i j}=x_{i j} \cdot p_{j}$. Also, $z_{j}=$ $\sum_{i} x_{i j}-1$ denotes the excess demand for good $j$. We sometimes index prices, spending, and demands by $t$ to indicate the relevant value at time $t$. Finally, we use a superscript of * to indicate an equilibrium value.

Bregman Divergence and Mirror Descent. Let $C$ be a compact and convex set. Given a differentiable convex function $h(\mathbf{x})$ with domain $C$, the Bregman divergence generated by kernel $h$ is denoted by $d_{h}$, and is defined as:

$$
d_{h}(\mathbf{x}, \mathbf{y})=h(\mathbf{x})-[h(\mathbf{y})+\langle\nabla h(\mathbf{y}), \mathbf{x}-\mathbf{y}\rangle], \forall \mathbf{x} \in C \text { and } \mathbf{y} \in \operatorname{rint}(C),
$$

where $\operatorname{rint}(C)$ is the relative interior of $C$. We note that, in general, $d_{h}$ is asymmetric, i.e., $d_{h}(\mathbf{x}, \mathbf{y}) \neq$ $d_{h}(\mathbf{y}, \mathbf{x})$. In this paper, we use the Kullback-Leibler or KL divergence extensively; it is the Bregman divergence generated by $h(\mathbf{x})=\sum_{j}\left(x_{j} \cdot \ln x_{j}-x_{j}\right)$. When $\sum_{j} x_{j}=\sum_{j} y_{j}$, the explicit formula is:

$$
\mathrm{KL}(\mathbf{x} \| \mathbf{y}):=\sum_{j} x_{j} \cdot \ln \frac{x_{j}}{y_{j}} .
$$

For the problem of minimizing a convex function $f(\mathbf{x})$ subject to $\mathbf{x} \in C$, the mirror descent method w.r.t. Bregman divergence $d_{h}$ is given by the following update rule:

$$
\mathbf{x}^{t+1}=\underset{\mathbf{x} \in C}{\arg \min }\left\{f\left(\mathbf{x}^{t}\right)+\left\langle\nabla f\left(\mathbf{x}^{t}\right), \mathbf{x}-\mathbf{x}^{t}\right\rangle+\frac{1}{\Gamma_{t}} \cdot d_{h}\left(\mathbf{x}, \mathbf{x}^{t}\right)\right\}
$$

where $\Gamma_{t}>0$, and may be dependent on $t$.

Proportional Response. For linear utility functions, Proportional Response is the dynamic given by the spending update rule:

$$
b_{i j}^{t+1}=e_{i} \cdot \frac{a_{i j} x_{i j}^{t}}{\sum_{k} a_{i k} x_{i k}^{t}}=e_{i} \cdot \frac{a_{i j} \frac{b_{i j}^{t}}{p_{j}^{t}}}{\sum_{k} a_{i k} \frac{b_{i k}^{t}}{p_{k}^{t}}} \text { with } p_{k}^{t}=\sum_{i} b_{i k}^{t} .
$$

For substitutes CES utilities, [50] generalized this rule to:

$$
b_{i j}^{t+1}=e_{i} \cdot \frac{a_{i j}\left(x_{i j}^{t}\right)^{\rho_{i}}}{\sum_{k} a_{i k}\left(x_{i k}^{t}\right)^{\rho_{i}}}=e_{i} \cdot \frac{a_{i j}\left(\frac{b_{i j}^{t}}{p_{j}^{t}}\right)^{\rho_{i}}}{\sum_{k} a_{i k}\left(\frac{b_{i k}^{t}}{p_{k}^{t}}\right)^{\rho_{i}}}
$$

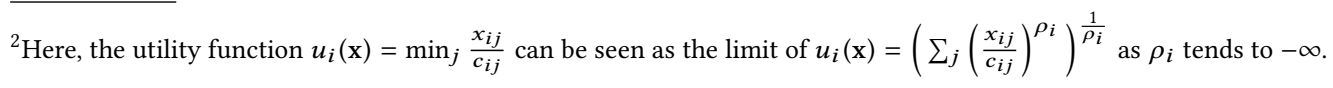


obtaining a linear convergence rate for the resulting dynamic, assuming $0<\rho_{i}<1$. The above rule has a natural distributed interpretation in the Fisher market setting: in each round, each buyer splits her spending on different goods in proportion to the values of $a_{i k}\left(x_{i k}^{t}\right)^{\rho_{i}}$. The seller of good $j$ then allocates the good to buyers in proportion to the spending received from each buyer.

\section{RESULTS}

\subsection{Proportional Response}

It is natural to seek to extend the Proportional Response rule (2) to the complementary domain, but this rule does not lead to convergent behavior in general. Set $\rho=-1$. Suppose there are two buyers and two items. Both buyers have the same preference for each item and the same budgets; i.e. $a_{11}=a_{12}=a_{21}=a_{22}=\frac{1}{2}$, and $e_{1}=e_{2}=1$. Initially, at time $t=0$, suppose that $b_{11}^{(0)}=\frac{1}{4}, b_{12}^{(0)}=\frac{3}{4}$, $b_{21}^{(0)}=\frac{3}{4}$, and $b_{22}^{(0)}=\frac{1}{4}$. A simple calculation shows that applying update rule (2) gives $b_{11}^{(1)}=\frac{3}{4}$, $b_{12}^{(1)}=\frac{1}{4}, b_{21}^{(1)}=\frac{1}{4}$, and $b_{22}^{(1)}=\frac{3}{4}$. So this simple example shows that in this setting, the spending will not converge to the market equilibrium; rather, it will cycle among 2 states.

Instead, we observe that in the substitutes domain, excluding Cobb-Douglas utilities, this rule is the mirror descent updating rule using the KL divergence for the following optimization problem.

$$
\begin{aligned}
& \min _{\mathbf{b}} \Phi(\mathbf{b})=-\sum_{i j} \frac{b_{i j}}{\rho_{i}} \log \frac{a_{i j}\left(b_{i j}\right)^{\rho_{i}-1}}{\left(\sum_{h} b_{h j}\right)^{\rho_{i}}} \\
& \text { subject to } \sum_{j} b_{i j}=e_{i} \text { for all } i, \text { and } b_{i j} \geq 0 \text { for all } i, j .
\end{aligned}
$$

We exclude Cobb-Douglas utilities, because as $\rho_{i} \rightarrow 0$ the corresponding term in $\Phi$ tends to $\infty$. When restricted to linear utilities, i.e. $\rho_{i}=1$ for all $i$, this is simply Shmyrev's convex program [44] for these markets.

In the complementary domain, the mirror descent updating rule for this function is:

$$
\begin{aligned}
& b_{i j}^{t+1}=e_{i} \cdot \frac{\left(\frac{a_{i j}}{\left(p_{j}^{t}\right)^{\rho_{i}}}\right)^{\frac{1}{1-\rho_{i}}}}{\sum_{k}\left(\frac{a_{i k}}{\left(p_{k}^{t}\right)^{\rho_{i}}}\right)^{\frac{1}{1-\rho_{i}}}} \text { for }-\infty<\rho_{i}<0, \quad \text { and } b_{i j}^{t+1}=e_{i} \cdot \frac{c_{i j} p_{j}^{t}}{\sum_{k} c_{i k} p_{k}^{t}} \text { for } \rho_{i}=-\infty, \\
& \text { where } p_{k}^{t}=\sum_{i} b_{i k}^{t} .
\end{aligned}
$$

Accordingly, we adopt this as the generalization of Proportional Response to the complementary domain. This rule can be easily implemented in the distributed environment of Fisher markets. In each round, each buyer only needs the prices computed in the previous round to compute its update. Thus, to implement the update rule, it suffices to have the sellers broadcast their prices by the end of each round.

Interestingly, this update rule is also the best response action to the current prices for each buyer. We note that this rule can be viewed as a tatonnement update if we define $x_{i j}^{t+1}=b_{i j}^{t+1} / p_{j}^{t}$, for then $p_{j}^{t+1}=\sum_{i} b_{i j}^{t+1}=\sum_{i} x_{i j}^{t+1} p_{j}^{t}=p_{j}^{t}\left(1+z_{j}^{t+1}\right)$. However, this is not the same rule as was used for the tatonnement analyzed in recent works regarding Fisher markets $[11,16]$.

For linear utilities, update rule (2) was analyzed in [2]. For the substitutes domain excluding linear utilities, a faster linear rate of convergence was shown in [50], but not based on considering the above optimization problem. To obtain a linear rate of convergence for an analysis based on 
optimizing $\Phi$ via a mirror descent with a KL Divergence, we introduce the notion of strong Bregman convexity. We also coin the term Bregman convexity for an analogous notion introduced in [2]).

Definition 1. The function $f$ is L-Bregman convex w.r.t. Bregman divergence $d_{h}$ if, for any $\mathrm{y} \in \operatorname{rint}(C)$ and $\mathrm{x} \in C$,

$$
f(\mathbf{y})+\langle\nabla f(\mathbf{y}), \mathbf{x}-\mathbf{y}\rangle \leq f(\mathbf{x}) \leq f(\mathbf{y})+\langle\nabla f(\mathbf{y}), \mathbf{x}-\mathbf{y}\rangle+L \cdot d_{h}(\mathbf{x}, \mathbf{y}) .
$$

The function $f$ is $(\sigma, L)$-strongly Bregman convex w.r.t. Bregman divergence $d_{h}$ if, $0<\sigma \leq L$, and, for any $\mathbf{y} \in \operatorname{rint}(C), \mathbf{x} \in C$,

$$
f(\mathbf{y})+\langle\nabla f(\mathbf{y}), \mathbf{x}-\mathbf{y}\rangle+\sigma \cdot d_{h}(\mathbf{x}, \mathbf{y}) \leq f(\mathbf{x}) \leq f(\mathbf{y})+\langle\nabla f(\mathbf{y}), \mathbf{x}-\mathbf{y}\rangle+L \cdot d_{h}(\mathbf{x}, \mathbf{y}) .
$$

If the direction of the inequalities and the signs on the $d_{h}(\mathbf{x}, \mathbf{y})$ terms are reversed, the function is said to be Bregman concave (or strongly Bregman concave respectively). ( $\operatorname{rint}(C)$ denotes the relative interior of $C$.)

Consider the update rule:

$$
\mathbf{x}^{t+1} \leftarrow \underset{\mathbf{y}}{\arg \min }\left\{\left\langle\nabla f\left(\mathbf{x}^{t}\right), \mathbf{y}-\mathbf{x}^{t}\right\rangle+L \cdot d_{h}\left(\mathbf{y}, \mathbf{x}^{t}\right)\right\} .
$$

THeorem 3.1. Suppose that $f$ is $(\sigma, L)$-strongly Bregman convex w.r.t. $d_{h}$. If update rule (4) is applied, then, for all $t \geq 1$,

$$
f\left(\mathbf{x}^{t}\right)-f\left(\mathbf{x}^{*}\right) \leq \frac{\sigma}{\left(\frac{L}{L-\sigma}\right)^{t}-1} \cdot d_{h}\left(\mathbf{x}^{*}, \mathbf{x}^{0}\right) .
$$

An analogous Theorem for $L$-Bregman convex functions was given in [2]:

Theorem 3.2. [2] Suppose $f$ is an L-Bregman convex function w.r.t. $d$, and $\mathbf{x}^{T}$ is the point reached after $T$ applications of the mirror descent update rule (4). Then,

$$
f\left(\mathbf{x}^{T}\right)-f\left(\mathbf{x}^{*}\right) \leq \frac{L \cdot d\left(\mathbf{x}^{*}, \mathbf{x}^{0}\right)}{T} .
$$

We show that in the CES substitutes domain, excluding linear utilities, $\Phi$ is a strongly Bregman convex function w.r.t. the KL-divergence on spending, thereby providing an alternative derivation of Zhang's result. In addition, in the CES complements domain, excluding Leontief utilities, $\Phi$ is a strongly Bregman concave function w.r.t. the KL divergence on spending, which yields a proof of linear convergence in this domain. These analyses are readily modified to give a $1 / T$ rate of convergence if we include respectively the linear and Leontief utilities. These results are made precise in the following theorem.

Theorem 3.3. Suppose buyers with substitutes utilities repeatedly update their spending using Proportional Response rule (2), and those with complementary utilities use rule (3). Then the potential function $\Phi$ converges to the market equilibrium as follows.

- If all buyers have substitutes CES utilities, then

$$
\Phi\left(\mathbf{b}^{T}\right)-\Phi\left(\mathbf{b}^{*}\right) \leq \frac{1}{T} \sum_{i} \frac{1}{\rho_{i}} \mathrm{KL}\left(b_{i}^{*} \| b_{i}^{0}\right) .
$$

- Suppose that in addition no buyer has a linear utility. Let $\sigma=\min _{i}\left\{1-\rho_{i}\right\}$. Then,

$$
\Phi\left(\mathbf{b}^{T}\right)-\Phi\left(\mathbf{b}^{*}\right) \leq \frac{\sigma(1-\sigma)^{T}}{1-(1-\sigma)^{T}} \sum_{i} \frac{1}{\rho_{i}} \mathrm{KL}\left(b_{i}^{*} \| b_{i}^{0}\right) .
$$


- If all buyers have complementary CES utilities, then $^{3}$

$$
\Phi\left(\mathbf{b}^{*}\right)-\Phi\left(\mathbf{b}^{T}\right) \leq \frac{1}{T} \sum_{i} \frac{\rho_{i}-1}{\rho_{i}} \mathrm{KL}\left(b_{i}^{*} \| b_{i}^{0}\right) .
$$

- Suppose that in addition no buyer has a Leontief utility. Let $\sigma=\min _{i}\left\{\frac{1}{1-\rho_{i}}\right\}$. Then,

$$
\Phi\left(\mathbf{b}^{*}\right)-\Phi\left(\mathbf{b}^{T}\right) \leq \frac{\sigma(1-\sigma)^{T}}{1-(1-\sigma)^{T}} \sum_{i} \frac{\rho_{i}-1}{\rho_{i}} \mathrm{KL}\left(b_{i}^{*} \| b_{i}^{0}\right) .
$$

The first two results are shown in Corollaries 6.4, 6.5, respectively, and the remaining results can be found in the full version of the paper. We also note that as shown in Lemma 13 in [2], if $b_{i j}=e_{i} / m$ for all $i$ and $j$, then $\mathrm{KL}\left(\mathbf{b}^{*}|| \mathbf{b}\right) \leq \log m n$, which provides a possibly more intuitive version of the above bounds.

Theorem 3.3 does not cover buyers with Cobb-Douglas utilities, because, as already noted, the terms in $\Phi$ for such buyers are equal to $\infty$. Note that these buyers always wish to allocate their spending in fixed proportions regardless of the prices. Thus, arguably, it would be natural for these buyers to always have the equilibrium spending. But even if this were not true initially, after one update this property would hold, and remain true henceforth. Thus the presence of these buyers would seem to have little effect on the convergence. Indeed, the above bounds hold with $\mathrm{KL}\left(\mathbf{b}_{i}^{*}|| \mathbf{b}_{i}^{0}\right)$ replaced by $\mathrm{KL}\left(\mathbf{b}_{i}^{*}|| \mathbf{b}_{i}^{1}\right)$ and $T$ replaced by $T-1$ on the RHS. But to obtain bounds in terms of $\mathrm{KL}\left(\mathbf{b}_{i}^{*}|| \mathbf{b}_{i}^{0}\right)$ appears to require substantially more effort; this analysis is given in the full paper. The rates of convergence are similar to those given in Theorem 3.3.

\subsection{Damped Proportional Response}

But what if we want to allow a mix of substitutes and complementary utilities? The difficulty we face is that the objective function $\Phi$ is no longer either convex or concave. Rather, if we fix the spending of the buyers with complementary utilities, the resulting restricted $\Phi$ is convex, while if we fix the spending of the buyers with substitutes utilities, the resulting restricted $\Phi$ is concave. As it happens, the equilibrium corresponds to a saddle point of the function $\Phi$. Also, a suitable dynamic will converge to this saddle point. To show this, we introduce a saddle-point convergence analysis. To this end, we define the following notion.

Definition 2. Function $f$ is $\left(L_{X}, L_{Y}\right)$-convex-concave w.r.t. the pair of Bregman divergences $\left(d_{g}, d_{h}\right)$, if it satisfies the following constraints.

(1) For fixed $\mathbf{y}, f(\cdot, \mathbf{y})$ is a convex function;

(2) For fixed $\mathbf{x}, f(\mathbf{x}, \cdot)$ is a concave function;

(3) There exist parameters $L_{X}, L_{Y}>0$ such that for any $\mathbf{x} \in X, \mathbf{x}^{\prime} \in X, \mathbf{y} \in Y$ and $\mathbf{y}^{\prime} \in Y$,

$$
-L_{Y} \cdot d_{h}\left(\mathbf{y}, \mathbf{y}^{\prime}\right) \stackrel{(a)}{\leq} f(\mathbf{x}, \mathbf{y})-f\left(\mathbf{x}^{\prime}, \mathbf{y}^{\prime}\right)-\left\langle\nabla f\left(\mathbf{x}^{\prime}, \mathbf{y}^{\prime}\right),(\mathbf{x}, \mathbf{y})-\left(\mathbf{x}^{\prime}, \mathbf{y}^{\prime}\right)\right\rangle \stackrel{(b)}{\leq} L_{X} \cdot d_{g}\left(\mathbf{x}, \mathbf{x}^{\prime}\right) .
$$

The saddle point is the "optimal" point of the convex-concave function, which is the minimum point in the $x$-direction and the maximum point in the $y$-direction, defined formally as follows.

Definition 3. $\left(x^{*}, y^{*}\right)$ is a saddle point of $f$ if and only if

$$
f\left(x, y^{*}\right) \geq f\left(x^{*}, y^{*}\right) \geq f\left(x^{*}, y\right) \text { for any } x \in X \text { and } y \in Y \text {. }
$$

${ }^{3}$ For $\rho_{i}=-\infty$, we define $\frac{\rho_{i}-1}{\rho_{i}}$ to equal 1 . 
Now consider the following update rule:

$$
\begin{aligned}
& \mathbf{x}^{t+1}=\arg \min _{\mathbf{x}}\left\{\left\langle\nabla_{x} f\left(\mathbf{x}^{t}, \mathbf{y}^{t}\right), \mathbf{x}-\mathbf{x}^{t}\right\rangle+2 L_{X} \cdot d_{g}\left(\mathbf{x}, \mathbf{x}^{t}\right)\right\} \\
& \mathbf{y}^{t+1}=\arg \min _{\mathbf{y}}\left\{\left\langle-\nabla_{\mathbf{y}} f\left(\mathbf{x}^{t}, \mathbf{y}^{t}\right), \mathbf{y}-\mathbf{y}^{t}\right\rangle+2 L_{Y} \cdot d_{h}\left(\mathbf{y}, \mathbf{y}^{t}\right)\right\} .
\end{aligned}
$$

We can then show an $O(1 / T)$ empirical rate of convergence, as stated in the next theorem.

THEOREM 3.4. Suppose that $f$ is $\left(L_{X}, L_{Y}\right)$-convex-concave, and there exists a saddle point $\left(\mathbf{x}^{*}, \mathbf{y}^{*}\right)$. In addition, suppose that $(\mathbf{x}, \mathbf{y})$ are updated according to (6). Then:

(i) $\sum_{t=1}^{T}\left(f\left(\mathbf{x}^{t}, \mathbf{y}^{*}\right)-f\left(\mathbf{x}^{*}, \mathbf{y}^{t}\right)\right) \leq 2 L_{X} \cdot d_{g}\left(\mathbf{x}^{*}, \mathbf{x}^{0}\right)+2 L_{Y} \cdot d_{h}\left(\mathbf{y}^{*}, \mathbf{y}^{0}\right)$.

Note that $f\left(\mathbf{x}^{t}, \mathbf{y}^{*}\right)-f\left(\mathbf{x}^{*}, \mathbf{y}^{t}\right) \geq 0$ since $f\left(\mathbf{x}^{t}, \mathbf{y}^{*}\right) \geq f\left(\mathbf{x}^{*}, \mathbf{y}^{*}\right) \geq f\left(\mathbf{x}^{*}, \mathbf{y}^{t}\right)$.

(ii) Also, if $\overline{\mathbf{x}}=\frac{1}{T} \sum_{t=1}^{T} \mathbf{x}^{t}$ and $\overline{\mathbf{y}}=\frac{1}{T} \sum_{t=1}^{T} \mathbf{y}^{t}$, then:

$$
f\left(\overline{\mathbf{x}}, \mathbf{y}^{*}\right)-f\left(\mathbf{x}^{*}, \overline{\mathbf{y}}\right) \leq \frac{1}{T}\left[2 L_{X} \cdot d_{g}\left(\mathbf{x}^{*}, \mathbf{x}^{0}\right)+2 L_{Y} \cdot d_{h}\left(\mathbf{y}^{*}, \mathbf{y}^{0}\right)\right] .
$$

Note that the second part of the theorem follows immediately from the first part because $f\left(\cdot, \mathbf{y}^{*}\right)$ is a convex function and $f\left(\mathbf{x}^{*}, \cdot\right)$ is a concave function.

The objective function $\Phi$ is $(1,1)$-convex-concave w.r.t. $d_{g}=\sum_{i: \rho_{i}>0} \frac{1}{\rho_{i}} \mathrm{KL}\left(b_{i} \| b_{i}^{\prime}\right)$ and $d_{h}=$ $\sum_{i: \infty<\rho_{i}<0} \frac{\rho_{i}-1}{\rho_{i}} \mathrm{KL}\left(b_{i} \| b_{i}^{\prime}\right)+\sum_{i: \rho_{i}=-\infty} \mathrm{KL}\left(b_{i} \| b_{i}^{\prime}\right)$. Consequently, we obtain an empirical $O(1 / T)$ rate of convergence for the following Damped Proportional Response update.

$b_{i j}^{t+1}=e_{i} \cdot \frac{\left[b_{i j}^{t} \cdot a_{i j}\left(\frac{b_{i j}^{t}}{p_{j}^{t}}\right)^{\rho_{i}}\right]^{\frac{1}{2}}}{\sum_{k}\left[b_{i k}^{t} \cdot a_{i k}\left(\frac{b_{i k}^{t}}{p_{k}^{t}}\right)^{\rho_{i}}\right]^{\frac{1}{2}}}$, for $\rho_{i}>0 ; b_{i j}^{t+1}=e_{i} \cdot \frac{\left[b_{i j}^{t} \cdot\left(\frac{a_{i j}}{\left(p_{j}^{t}\right)^{\rho_{i}}}\right)^{\frac{1}{1-\rho_{i}}}\right]^{\frac{1}{2}}}{\sum_{k}\left[b_{i k}^{t} \cdot\left(\frac{a_{i k}}{\left(p_{k}^{t}\right)^{\rho_{i}}}\right)^{\frac{1}{1-\rho_{i}}}\right]^{\frac{1}{2}}}$, for $-\infty<\rho_{i}<0 ;$

$b_{i j}^{t+1}=e_{i} \cdot \frac{\left[b_{i j}^{t} \cdot\left(\frac{c_{i j}}{p_{j}^{t}}\right)^{-1}\right]^{\frac{1}{2}}}{\sum_{k}\left[b_{i k}^{t} \cdot\left(\frac{c_{i k}}{p_{k}^{t}}\right)^{-1}\right]^{\frac{1}{2}}}$, for $\rho_{i}=-\infty ;$

We say it is damped because the update rule uses the geometric mean of the current value and the standard Proportional Response update.

A natural question is whether a linear convergence rate is possible if the linear and Leontief utilities are excluded. The answer is yes, and to obtain this we need a stronger condition on the convex-concave objective function, as given in the following definition.

Definition 4. $f$ is a $\left(\sigma_{X}, \sigma_{Y}, L_{X}, L_{Y}\right)$-strongly Bregman convex-concave function, w.r.t. Bregman divergences $d_{g}, d_{h}$, if, for all $\mathbf{x} \in X, \mathbf{x}^{\prime} \in X, \mathbf{y} \in Y$, and $\mathbf{y}^{\prime} \in Y$, function $f$ satisfies:

$$
\begin{aligned}
& -L_{Y} \cdot d_{h}\left(\mathbf{y}, \mathbf{y}^{\prime}\right)+\sigma_{X} \cdot d_{g}\left(\mathbf{x}, \mathbf{x}^{\prime}\right) \leq f(\mathbf{x}, \mathbf{y})-f\left(\mathbf{x}^{\prime}, \mathbf{y}^{\prime}\right)-\left\langle\nabla f\left(\mathbf{x}^{\prime}, \mathbf{y}^{\prime}\right),(\mathbf{x}, \mathbf{y})-\left(\mathbf{x}^{\prime}, \mathbf{y}^{\prime}\right)\right\rangle \\
& \leq L_{X} \cdot d_{g}\left(\mathbf{x}, \mathbf{x}^{\prime}\right)-\sigma_{Y} \cdot d_{h}\left(\mathbf{y}, \mathbf{y}^{\prime}\right) .
\end{aligned}
$$

THEOREM 3.5. If $f$ is a $\left(\sigma_{X}, \sigma_{Y}, L_{X}, L_{Y}\right)$-strongly Bregman convex-concave function w.r.t $d_{g}$ and $d_{h}$, and there exists a saddle point, then update rule (6) converges to the saddle point with a linear convergence rate:

$$
\left(f\left(\mathbf{x}^{T}, \mathbf{y}^{*}\right)-f\left(\mathbf{x}^{*}, \mathbf{y}^{T}\right)\right) \leq\left(1-\frac{\min \left\{\frac{\sigma_{X}}{L_{X}}, \frac{\sigma_{Y}}{L_{Y}}\right\}}{2}\right)^{T-1}\left(\left(2 L_{X}-\sigma_{X}\right) d_{g}\left(\mathbf{x}^{*}, \mathbf{x}^{0}\right)+\left(2 L_{Y}-\sigma_{Y}\right) d_{h}\left(\mathbf{y}^{*}, \mathbf{y}^{0}\right)\right) .
$$


$\Phi$ is $\left(\min _{i: \rho_{i}>0}\left\{1-\rho_{i}\right\}, \min _{i: \rho_{i}<0}\left\{\frac{1}{1-\rho_{i}}\right\}, 1,1\right)$-strongly Bregman convex-concave, and thus we can deduce that the damped Proportional Response achieves a linear convergence rate if linear and Leontief utilities are excluded.

As before, the above results exclude Cobb-Douglas utilities.

Arguably, the buyers with Cobb-Douglas utilities should always have the equilibrium spending, or failing that, should immediately update to this spending. But for mathematical consistency, we suppose they are performing the same type of damped update as the other buyers. In this case, our previous potential function can't be used when we include Cobb-Douglas utility functions. We now need to include a term in the potential function for each buyer with a Cobb-Douglas utility as their spending keeps changing.

We will need the following notation. Let $\mathbf{b}_{>0}, \mathbf{b}_{=0}$, and $\mathbf{b}_{<0}$ denote the spending of those buyers with $\rho_{i}>0, \rho_{i}=0$, and $\rho_{i}<0$, respectively. Accordingly, we will write $\Phi(\mathbf{b})=\Phi\left(\mathbf{b}_{>0}, \mathbf{b}_{=0}, \mathbf{b}_{<0}\right)$. The resulting function is still convex in $\mathbf{b}_{>0}$ and concave in $\mathbf{b}_{<0}$. The construction is given in the full paper. We note that the update rule for the buyers with $\rho_{i}=0$ is given by:

$$
b_{i j}^{t+1}=e_{i} \cdot \frac{\left[b_{i j}^{t} \cdot a_{i j}^{t}\right]^{\frac{1}{2}}}{\sum_{k}\left[b_{i k}^{t} \cdot a_{i k}^{t}\right]^{\frac{1}{2}}}, \quad \text { for } \rho_{i}=0 .
$$

Theorem 3.6. Suppose buyers repeatedly update their spending using the Damped Proportional Response rule (7). Then

$$
\mathrm{KL}\left(\mathbf{b}_{=0}^{T} \| \mathbf{b}_{=0}^{*}\right) \leq \frac{1}{2^{T}} \mathrm{KL}\left(\mathbf{b}_{=0}^{0} \| \mathbf{b}_{=0}^{*}\right),
$$

and the potential function $\Phi$ converges to the market equilibrium as follows:

$$
\begin{aligned}
i . & \sum_{t=1}^{T}\left[\Phi\left(\mathbf{b}_{>0}^{t}, \mathbf{b}_{=0}^{*}, \mathbf{b}_{<0}^{*}\right)-\Phi\left(\mathbf{b}_{>0}^{*}, \mathbf{b}_{=0}^{*}, \mathbf{b}_{<0}^{t}\right)\right] \\
& \leq 4 \sum_{i: \rho_{i}=0} \mathrm{KL}\left(\mathbf{b}_{i}^{*} \| \mathbf{b}_{i}^{0}\right)+\sum_{i:-\infty<\rho_{i}<0} \frac{2\left(\rho_{i}-1\right)}{\rho_{i}} \mathrm{KL}\left(\mathbf{b}_{i}^{*} \| \mathbf{b}_{i}^{0}\right)+\sum_{i: \rho_{i}>0} \frac{2}{\rho_{i}} \mathrm{KL}\left(\mathbf{b}_{i}^{*} \| \mathbf{b}_{i}^{0}\right)+\sum_{i: \rho_{i}=-\infty} \mathrm{KL}\left(\mathbf{b}_{i}^{*} \| \mathbf{b}_{i}^{0}\right) .
\end{aligned}
$$

ii. If in addition no buyer has a linear or Leontief utility,

$$
\begin{aligned}
\text { Let } \sigma=\min \left\{\min _{i: \rho_{i}>0}\left\{\frac{2}{1+\rho_{i}}\right\}\right. & \left.\left., \min _{i: \rho_{i}<0}\left\{\frac{2\left(\rho_{i}-1\right)}{2 \rho_{i}-1}\right\}\right\} \quad \text { (so } 1<\sigma<2\right) . \text { Then, } \\
\Phi\left(\mathbf{b}_{>0}^{T}, \mathbf{b}_{=0}^{*}, \mathbf{b}_{<0}^{*}\right)-\Phi\left(\mathbf{b}_{>0}^{*}, \mathbf{b}_{=0}^{*}, \mathbf{b}_{<0}^{T}\right) \leq & \frac{1}{\sigma^{T-1}}\left[\frac{4}{2-\sigma} \sum_{i: \rho_{i}=0} \mathrm{KL}\left(\mathbf{b}_{i}^{*} \| \mathbf{b}_{i}^{0}\right)\right. \\
& \left.+\sum_{i: \rho_{i}<0} \frac{2 \rho_{i}-1}{\rho_{i}} \mathrm{KL}\left(\mathbf{b}_{i}^{*} \| \mathbf{b}_{i}^{0}\right)+\sum_{i: \rho_{i}>0} \frac{1+\rho_{i}}{\rho_{i}} \mathrm{KL}\left(\mathbf{b}_{i}^{*} \| \mathbf{b}_{i}^{0}\right)\right] .
\end{aligned}
$$

Weaker bounds are shown in Corollaries 8.2 and 8.3, respectively. The complete proof is given in the full paper. 


\section{RELATED WORK}

The concept of a market equilibrium was first proposed by Walras [48] along with a description of the tatonnement process. Since then, studies of market equilibria and tatonnement have received much attention in economics, operations research, and most recently in computer science. A fairly recent account of the classic perspective in economics is given in [34].

Computer scientists, beginning with the work by Deng et al. [19], showed that computing equilibria was a hard problem in general; see also [18, 41]. This led to much work on polynomial time algorithms for restricted classes of markets, e.g. [13, 20, 21, 25].

The Eisenberg-Gale program for the case of linear utilities was formulated in [24] and then generalized to homothetic functions in [23]; further generalizations were given in [28]. The maxima of these convex programs correspond to the equilibria of the corresponding markets. In particular, when buyer or agent utilities are homothetic, the optimum of the Eisenberg-Gale program corresponds to the optimum Nash Social Welfare; interestingly, this optimum also appears to provide good outcomes when apportioning indivisible goods [4, 5]. Recently, Cole et al. [15] identified another variant of the Eisenberg-Gale program that captured the best currently-known polynomial-time approximate solution for the indivisible setting.

The analysis most similar to ours is the one in [2] which considers convex functions that obey a constraint which we name $L$-Bregman convexity w.r.t. a Bregman divergence (see Definition 1 ). Our work generalizes this notion substantially.

The earliest analyses of tatonnement showed convergence in exchange economies with gross substitutes utilities, first for continuous updating [1] and then for discrete updates [45], but it was shown to diverge in general [43]. Recent works have analyzed its convergence properties in specific markets, primarily Fisher markets $[11,12,16]$. Cheung et al. [11] showed that tatonnement is equivalent to coordinate descent on a convex function for several classes of Fisher markets, and consequently that a suitable tatonnement converges toward the market equilibrium in two classes of markets: complementary-CES Fisher markets and Leontief Fisher markets.

Other dynamics have been considered. In particular, Dvijotham et al. [22] study sellers best responding in a setting in which they form beliefs about other sellers' strategies. They obtain linear convergence in Fisher markets where buyers have weak gross substitutes utilities. In the context of network flow control, Low and Lapsley [32] adopted an optimization approach to derive a dynamic protocol where both prices (of links) and flow demands of agents are updated, and showed that the protocol converges to a social-welfare maximizing state. The update rules (2), (3) look quite similar to a game-learning dynamic called log-linear learning $[3,33]$ (by suitably viewing spendings as probability densities), but due to different contexts (games vs. markets), the actual behaviors and the analyses have significant qualitative differences.

Convex-concave saddle-point problems can be reduced to non-smooth convex minimization problems, for which algorithms yielding $O(1 / \sqrt{T})$ convergence rate exist. Its wide applications (e.g., to two-person zero-sum game equilibrium) have motivated exploration of properties of the underlying function which support faster converging algorithms [36-39, 42]. In this paper, we present a new property and a simple algorithm which yields an $O(1 / T)$ empirical convergence rate. In our opinion, its analysis is quite simple, which may well open the door to further exploration. Indeed, we have taken such a step by presenting a variant of our new property for which the same algorithm yields a linear point-wise convergence rate. 


\section{LINEAR CONVERGENCE WITH STRONG BREGMAN CONVEXITY}

Our proof will use the following lemmas.

LEMMA 5.1. [6] If $\mathbf{x}^{+}$is the optimal point for the optimization problem:

$$
\begin{array}{ll}
\text { minimize } & g(\mathbf{x})+d(\mathbf{x}, \mathbf{y}) \\
\text { subject to } & \mathbf{x} \in C,
\end{array}
$$

where $C$ is a compact convex set, then, for any $\mathbf{x} \in C$,

$$
g\left(\mathbf{x}^{+}\right)+d\left(\mathbf{x}^{+}, \mathbf{y}\right)+d\left(\mathbf{x}, \mathbf{x}^{+}\right) \leq g(\mathbf{x})+d(\mathbf{x}, \mathbf{y}) .
$$

LEMMA 5.2. [2] Suppose that $f$ is a L-Bregman convex function w.r.t. $d\left(\mathbf{x}, \mathbf{x}^{\prime}\right)$, and $\mathbf{x}^{t}$ and $\mathbf{x}^{t+1}$ are the points reached after $t$ and $t+1$ applications of the mirror descent update rule (4). Then

$$
f\left(\mathbf{x}^{t+1}\right) \leq f\left(\mathbf{x}^{t}\right) .
$$

Proof of Theorem 3.1. By Lemma 5.1 with $\mathbf{y}=\mathbf{x}^{t}, \mathbf{x}^{+}=\mathbf{x}^{t+1}$, and $\mathbf{x}=\mathbf{x}^{*}$,

$\left\langle\nabla f\left(\mathbf{x}^{t}\right), \mathbf{x}^{t+1}-\mathbf{x}^{t}\right\rangle+L \cdot d_{h}\left(\mathbf{x}^{t+1}, \mathbf{x}^{t}\right) \leq\left\langle\nabla f\left(\mathbf{x}^{t}\right), \mathbf{x}^{*}-\mathbf{x}^{t}\right\rangle+L \cdot\left[d_{h}\left(\mathbf{x}^{*}, \mathbf{x}^{t}\right)-d_{h}\left(\mathbf{x}^{*}, \mathbf{x}^{t+1}\right)\right]$.

By strong Bregman-convexity, with $\mathbf{y}=\mathbf{x}^{t}$, and $\mathbf{x}=\mathbf{x}^{t+1}$,

$$
\left\langle\nabla f\left(\mathbf{x}^{t}\right), \mathbf{x}^{t+1}-\mathbf{x}^{t}\right\rangle+L \cdot d_{h}\left(\mathbf{x}^{t+1}, \mathbf{x}^{t}\right) \geq f\left(\mathbf{x}^{t+1}\right)-f\left(\mathbf{x}^{t}\right)
$$

and with $\mathbf{y}=\mathbf{x}^{t}$ and $\mathbf{x}=\mathbf{x}^{*}$,

$$
\nabla f\left(\mathbf{x}^{t}\right) \cdot\left(\mathbf{x}^{*}-\mathbf{x}^{t}\right) \leq f\left(\mathbf{x}^{*}\right)-f\left(\mathbf{x}^{t}\right)-\sigma \cdot d_{h}\left(\mathbf{x}^{*}, \mathbf{x}^{t}\right) .
$$

Combining (9), (10), and (11), gives, for $t \geq 0$,

$$
f\left(\mathbf{x}^{t+1}\right)-f\left(\mathbf{x}^{*}\right) \leq(L-\sigma) \cdot d_{h}\left(\mathbf{x}^{*}, \mathbf{x}^{t}\right)-L \cdot d_{h}\left(\mathbf{x}^{*}, \mathbf{x}^{t+1}\right) .
$$

On multiplying both sides of the above inequality by $\left(\frac{L}{L-\sigma}\right)^{t}$, and then summing over $0 \leq t<T$, the RHS becomes a telescoping sum, and hence

$$
\sum_{t=0}^{T-1}\left(\frac{L}{L-\sigma}\right)^{t} \cdot\left[f\left(\mathbf{x}^{t+1}\right)-f\left(\mathbf{x}^{*}\right)\right] \leq(L-\sigma) \cdot d_{h}\left(\mathbf{x}^{*}, \mathbf{x}^{0}\right)
$$

By Lemma 5.2, $f\left(\mathbf{x}^{t+1}\right) \leq f\left(\mathbf{x}^{t}\right)$; thus:

$\frac{L-\sigma}{\sigma} \cdot\left[\left(\frac{L}{L-\sigma}\right)^{T}-1\right] \cdot\left[f\left(\mathbf{x}^{T}\right)-f\left(\mathbf{x}^{*}\right)\right]=\left(\sum_{t=0}^{T-1}\left(\frac{L}{L-\sigma}\right)^{t}\right) \cdot\left[f\left(\mathbf{x}^{T}\right)-f\left(\mathbf{x}^{*}\right)\right] \leq(L-\sigma) \cdot d_{h}\left(\mathbf{x}^{*}, \mathbf{x}^{0}\right)$, and the result follows.

\section{CONVERGENCE OF PROPORTIONAL RESPONSE}

We consider the following potential function:

$$
\begin{aligned}
& p_{j}(\mathbf{b})=\sum_{i} b_{i j}, \\
& \Phi(\mathbf{b})=-\sum_{i: \rho_{i} \neq\{0,-\infty\}} \frac{1}{\rho_{i}} \sum_{j} b_{i j} \log \frac{a_{i j} b_{i j}^{\rho_{i}-1}}{\left[p_{j}(\mathbf{b})\right]^{\rho_{i}}}-\sum_{i: \rho_{i}=-\infty} \sum_{j} b_{i j} \log \frac{b_{i j}}{c_{i j} p_{j}(\mathbf{b})} .
\end{aligned}
$$


For those $i$ for which $\rho_{i} \neq-\infty$,

$$
\begin{aligned}
\nabla_{b_{i j}} \Phi(\mathbf{b}) & =-\frac{1}{\rho_{i}} \log a_{i j}-\frac{\rho_{i}-1}{\rho_{i}}\left(\log b_{i j}+1\right)+\log p_{j}(\mathbf{b})+\sum_{h} b_{h j} \frac{1}{p_{j}(\mathbf{b})} \\
& =\frac{1}{\rho_{i}}\left(1-\log \frac{a_{i j} b_{i j}^{\rho_{i}-1}}{p_{j}^{\rho_{i}}(\mathbf{b})}\right) ;
\end{aligned}
$$

and for those $i$ for which $\rho_{i}=-\infty, \quad \nabla_{b_{i j}} \Phi(\mathbf{b})=-\log \frac{b_{i j}}{c_{i j} p_{j}}$.

We deduce:

LEMMA 6.1.

$\sum_{i: \rho_{i} \neq-\infty} \frac{1-\rho_{i}}{\rho_{i}} \mathrm{KL}\left(\mathbf{b}_{i} \| \mathbf{b}_{i}^{\prime}\right)-\sum_{i: \rho_{i}=-\infty} \mathrm{KL}\left(\mathbf{b}_{i} \| \mathbf{b}_{i}^{\prime}\right) \leq \Phi(\mathbf{b})-\Phi\left(\mathbf{b}^{\prime}\right)-\left\langle\nabla \Phi\left(\mathbf{b}^{\prime}\right), \mathbf{b}-\mathbf{b}^{\prime}\right\rangle \leq \sum_{i: \rho_{i} \neq-\infty} \frac{1}{\rho_{i}} \mathrm{KL}\left(\mathbf{b}_{i} \| \mathbf{b}_{i}^{\prime}\right)$.

PROOF.

$$
\begin{aligned}
\Phi(\mathbf{b})-\Phi\left(\mathbf{b}^{\prime}\right)-\left\langle\nabla \Phi\left(\mathbf{b}^{\prime}\right), \mathbf{b}-\mathbf{b}^{\prime}\right\rangle & =-\sum_{i: \rho_{i} \neq\{0,-\infty\}} \frac{\rho_{i}-1}{\rho_{i}} \mathrm{KL}\left(\mathbf{b}_{i} \| \mathbf{b}_{i}^{\prime}\right)-\sum_{i: \rho_{i}=-\infty} \mathrm{KL}\left(\mathbf{b}_{i} \| \mathbf{b}_{i}^{\prime}\right)+\mathrm{KL}\left(\mathbf{p} \| \mathbf{p}^{\prime}\right) \\
& =\sum_{i: \rho_{i} \neq-\infty} \frac{1}{\rho_{i}} \mathrm{KL}\left(\mathbf{b}_{i} \| \mathbf{b}_{i}^{\prime}\right)-\left(\sum_{i} \mathrm{KL}\left(\mathbf{b}_{i} \| \mathbf{b}_{i}^{\prime}\right)-\mathrm{KL}\left(\mathbf{p} \| \mathbf{p}^{\prime}\right)\right) .
\end{aligned}
$$

Since $\sum_{i} \mathrm{KL}\left(\mathbf{b}_{i} \| \mathbf{b}_{i}^{\prime}\right) \geq \mathrm{KL}\left(\mathbf{p} \| \mathbf{p}^{\prime}\right)$, the result follows.

The Substitutes Domain. The following lemma states the equivalence between mirror descent and proportional response in the substitutes domain; it follow readily from the definition of $\mathbf{b}^{t+1}$ for Proportional Response (given by (2)).

Lemma 6.2. For buyers with CES substitutes utilities, the Proportional Response update is the same as the mirror descent update, given by:

$$
\mathbf{b}_{i}^{t+1}=\arg \min _{\mathbf{b}_{i}: \sum_{j} b_{i j}=e_{i}}\left\{\left\langle\nabla_{\mathbf{b}_{i}} \Phi\left(\mathbf{b}^{t}\right), \mathbf{b}_{i}-\mathbf{b}_{i}^{t}\right\rangle+\frac{1}{\rho_{i}} \mathrm{KL}\left(\mathbf{b}_{i} \| \mathbf{b}_{i}^{t}\right)\right\} .
$$

The next lemma states several properties of the potential function in the substitutes domain.

LEMMA 6.3. [i.]

(1) If $\rho_{i}>0$ for all $i$, then $\Phi$ is a 1-Bregman convex function w.r.t. $\sum_{i} \frac{1}{\rho_{i}} \mathrm{KL}\left(\mathbf{b}_{i} \| \mathbf{b}_{i}^{\prime}\right)$;

(2) if $0<\rho_{i}<1$ for all $i$, then $\Phi$ is a $\left(\min _{i}\left\{1-\rho_{i}\right\}\right.$, 1$)$-strong Bregman convex function w.r.t. $\sum_{i} \frac{1}{\rho_{i}} \mathrm{KL}\left(\mathbf{b}_{i} \| \mathbf{b}_{i}^{\prime}\right)$;

(3) $\mathbf{b}$ is the spending at the market equilibrium if and only if $\mathbf{b}$ is the minimum point of $\Phi$.

Proof. The first two claims follow from Lemma 6.1 with a little calculation. We show the third claim for CES utilities, leaving the cases of linear utilities to the full paper.

First, we note that the update rule will leave $\mathbf{b}$ unchanged exactly if $\mathbf{b}=\mathbf{b}^{*}$. This implies that for every $i$, the values $a_{i j}\left(b_{i j}\right)^{\rho_{i}-1} / p_{j}^{\rho_{i}}$ are the same at a minimum of $\Phi$, for if $\mathbf{b}^{t}$ is optimal then $\mathbf{b}^{t+1}=\mathbf{b}^{t}$, and so for all $j$ the multipliers on $b_{i j}^{t}$ are the same, and these multipliers are $a_{i j}\left(b_{i j}\right)^{\rho_{i}-1} / p_{j}^{\rho_{i}}$. But this is exactly the condition that holds when buyer $i$ is maximizing her utility 
at prices p: $a_{i j}\left(x_{i j}\right)^{\rho_{i}-1} / p_{j}$ are the same for all $j$, and since $x_{i j}=b_{i j} / p_{j}$, this means $a_{i j}\left(b_{i j}\right)^{\rho_{i}-1} / p_{j}^{\rho_{i}}$ are the same for all $j$.

Let $\mathbf{b}^{*}$ be the spending at some market equilibrium. Applying Theorem 3.2 yields:

Corollary 6.4.

$$
\Phi\left(\mathbf{b}^{T}\right)-\Phi\left(\mathbf{b}^{*}\right) \leq \frac{1}{T} \sum_{i} \frac{1}{\rho_{i}} \mathrm{KL}\left(\mathbf{b}_{i}^{*} \| \mathbf{b}_{i}^{0}\right) .
$$

Furthermore, if there is no buyer with a linear utility function, then, applying Theorem 3.1 yields:

Corollary 6.5. Let $\sigma=\min _{i}\left\{1-\rho_{i}\right\}$. Then,

$$
\Phi\left(\mathbf{b}^{T}\right)-\Phi\left(\mathbf{b}^{*}\right) \leq \frac{\sigma(1-\sigma)^{T}}{1-(1-\sigma)^{T}} \sum_{i} \frac{1}{\rho_{i}} \mathrm{KL}\left(\mathbf{b}_{i}^{*} \| \mathbf{b}_{i}^{0}\right) .
$$

We now explain how to recover Zhang's bound [50]. From (12),

$$
\sum_{i} \frac{1}{\rho_{i}} \mathrm{KL}\left(\mathbf{b}_{i}^{*} \| \mathbf{b}_{i}^{t}\right) \leq \frac{L-\sigma}{L} \sum_{i} \frac{1}{\rho_{i}} \mathrm{KL}\left(\mathbf{b}_{i}^{*} \| \mathbf{b}_{i}^{t-1}\right) \leq\left(\frac{L-\sigma}{L}\right)^{t} \sum_{i} \frac{1}{\rho_{i}} \mathrm{KL}\left(\mathbf{b}_{i}^{*} \| \mathbf{b}_{i}^{0}\right)=\left(\max _{i} \rho_{i}\right)^{t} \sum_{i} \frac{1}{\rho_{i}} \mathrm{KL}\left(\mathbf{b}_{i}^{*} \| \mathbf{b}_{i}^{0}\right) .
$$

In [50], $\phi(t)$ is used to denote $\sum_{i} \frac{1}{\rho_{i}} \mathrm{KL}\left(\mathbf{b}_{i}^{*} \| \mathbf{b}_{i}^{t}\right)$. We have obtained the exact same bound on $\sum_{i} \frac{1}{\rho_{i}} \mathrm{KL}\left(\mathbf{b}_{i}^{*}|| \mathbf{b}_{i}^{t}\right)$ as in [50], and thus can deduce the identical convergence rate.

The Complementary Domain. The analysis here is similar to that for the substitutes domain and is given in the full version of the paper.

\section{SADDLE POINT ANALYSIS}

Proof of Theorem 3.4. Recall that $\mathbf{x}^{t+1}=\arg \min _{\mathbf{x} \in X}\left\{\left\langle\nabla_{\mathbf{x}} f\left(\mathbf{x}^{t}, \mathbf{y}^{t}\right), \mathbf{x}-\mathbf{x}^{t}\right\rangle+2 L_{X} d_{g}\left(\mathbf{x}, \mathbf{x}^{t}\right)\right\}$. Applying Lemma 5.1 with $\mathbf{x}=\mathbf{x}^{*}, d(\cdot, \cdot)=2 L_{X} d_{g}(\cdot, \cdot), \mathbf{y}=\mathbf{x}^{t}, \mathbf{x}^{+}=\mathbf{x}^{t+1}$, and $g(\mathbf{x})=\left\langle\nabla_{x} f\left(\mathbf{x}^{t}, \mathbf{y}^{t}\right)\right\rangle, \mathbf{x}-$ $\left.\mathbf{x}^{t}\right\rangle$ gives

$\left\langle\nabla_{x} f\left(\mathbf{x}^{t}, \mathbf{y}^{t}\right), \mathbf{x}^{t+1}-\mathbf{x}^{t}\right\rangle+2 L_{X} d_{g}\left(\mathbf{x}^{t+1}, \mathbf{x}^{t}\right) \leq\left\langle\nabla_{x} f\left(\mathbf{x}^{t}, \mathbf{y}^{t}\right), \mathbf{x}^{*}-\mathbf{x}^{t}\right\rangle+2 L_{X} d_{g}\left(\mathbf{x}^{*}, \mathbf{x}^{t}\right)-2 L_{X} d_{g}\left(\mathbf{x}^{*}, \mathbf{x}^{t+1}\right)$.

This is equivalent to

$$
\begin{aligned}
& \underbrace{f\left(\mathbf{x}^{t}, \mathbf{y}^{t}\right)+\left\langle\nabla f\left(\mathbf{x}^{t}, \mathbf{y}^{t}\right),\left(\mathbf{x}^{t+1}, \mathbf{y}^{t+1}\right)-\left(\mathbf{x}^{t}, \mathbf{y}^{t}\right)\right\rangle+2 L_{X} d_{g}\left(\mathbf{x}^{t+1}, \mathbf{x}^{t}\right)}_{\text {RHS }} \\
& \quad \leq \underbrace{f\left(\mathbf{x}^{t}, \mathbf{y}^{t}\right)+\left\langle\nabla f\left(\mathbf{x}^{t}, \mathbf{y}^{t}\right),\left(\mathbf{x}^{*}, \mathbf{y}^{t+1}\right)-\left(\mathbf{x}^{t}, \mathbf{y}^{t}\right)\right\rangle+2 L_{X} d_{g}\left(\mathbf{x}^{*}, \mathbf{x}^{t}\right)-2 L_{X} d_{g}\left(\mathbf{x}^{*}, \mathbf{x}^{t+1}\right)}_{\text {LHS }} .
\end{aligned}
$$

Since $f$ is $\left(L_{X}, L_{y}\right)$-convex-concave, the third property - see (5) - gives:

$$
\begin{aligned}
f\left(\mathbf{x}^{t+1}, \mathbf{y}^{t+1}\right)+L_{X} d_{g}\left(\mathbf{x}^{t+1}, \mathbf{x}^{t}\right) \stackrel{(1)}{\leq} \text { LHS } \leq \text { RHS } \\
\quad \stackrel{(2)}{\leq} f\left(\mathbf{x}^{*}, \mathbf{y}^{t+1}\right)+L_{Y} d_{h}\left(\mathbf{y}^{t+1}, \mathbf{y}^{t}\right)+2 L_{X} d_{g}\left(\mathbf{x}^{*}, \mathbf{x}^{t}\right)-2 L_{X} d_{g}\left(\mathbf{x}^{*}, \mathbf{x}^{t+1}\right),
\end{aligned}
$$

where (1) is deduced from $(b)$ in Definition 2 with $\left(x^{\prime}, y^{\prime}\right)=\left(\mathbf{x}^{t}, \mathbf{y}^{t}\right)$ and (2) is deduced from $(a)$ in Definition 2 with $\left(x^{\prime}, y^{\prime}\right)=\left(\mathbf{x}^{t}, \mathbf{y}^{t}\right)$ and $(x, y)=\left(\mathbf{x}^{*}, \mathbf{y}^{t+1}\right)$. 
Now, let's consider $-f(x, y)$ and $\mathbf{y}^{t+1}=\arg \min _{\mathbf{y} \in Y}\left\{\left\langle-\nabla_{\mathbf{y}} f\left(\mathbf{x}^{t}, \mathbf{y}^{t}\right), \mathbf{y}-\mathbf{y}^{t}\right\rangle+2 L_{Y} d_{h}\left(\mathbf{y}, \mathbf{y}^{t}\right)\right\}$. Using a similar argument, we obtain:

$-f\left(\mathbf{x}^{t+1}, \mathbf{y}^{t+1}\right)+L_{Y} d_{h}\left(\mathbf{y}^{t+1}, \mathbf{y}^{t}\right) \leq-f\left(\mathbf{x}^{t+1}, \mathbf{y}^{*}\right)+L_{X} d_{g}\left(\mathbf{x}^{t+1}, \mathbf{x}^{t}\right)+2 L_{Y} d_{h}\left(\mathbf{y}^{*}, \mathbf{y}^{t}\right)-2 L_{Y} d_{h}\left(\mathbf{y}^{*}, \mathbf{y}^{t+1}\right)$.

Adding these two inequalities gives:

$f\left(\mathbf{x}^{t+1}, \mathbf{y}^{*}\right)-f\left(\mathbf{x}^{*}, \mathbf{y}^{t+1}\right) \leq 2 L_{X} d_{g}\left(\mathbf{x}^{*}, \mathbf{x}^{t}\right)+2 L_{Y} d_{h}\left(\mathbf{y}^{*}, \mathbf{y}^{t}\right)-2 L_{X} d_{g}\left(\mathbf{x}^{*}, \mathbf{x}^{t+1}\right)-2 L_{Y} d_{h}\left(\mathbf{y}^{*}, \mathbf{y}^{t+1}\right)$.

Summing over $t$ yields: $\quad \sum_{t=1}^{T}\left(f\left(\mathbf{x}^{t}, \mathbf{y}^{*}\right)-f\left(\mathbf{x}^{*}, \mathbf{y}^{t}\right)\right) \leq 2 L_{X} d_{g}\left(\mathbf{x}^{*}, \mathbf{x}^{0}\right)+2 L_{Y} d_{h}\left(\mathbf{y}^{*}, \mathbf{y}^{0}\right)$.

Proof of Theorem 3.5. Using (8) instead of (5), we deduce the following from (13) instead of (14):

$f\left(\mathbf{x}^{t+1}, \mathbf{y}^{t+1}\right)+L_{X} d_{g}\left(\mathbf{x}^{t+1}, \mathbf{x}^{t}\right) \leq f\left(\mathbf{x}^{*}, \mathbf{y}^{t+1}\right)+L_{Y} d_{h}\left(\mathbf{y}^{t+1}, \mathbf{y}^{t}\right)+\left(2 L_{X}-\sigma_{X}\right) d_{g}\left(\mathbf{x}^{*}, \mathbf{x}^{t}\right)-2 L_{X} d_{g}\left(\mathbf{x}^{*}, \mathbf{x}^{t+1}\right)$.

Also, (15) is replaced by:

$-f\left(\mathbf{x}^{t+1}, \mathbf{y}^{t+1}\right)+L_{Y} d_{h}\left(\mathbf{y}^{t+1}, \mathbf{y}^{t}\right) \leq-f\left(\mathbf{x}^{t+1}, \mathbf{y}^{*}\right)+L_{X} d_{g}\left(\mathbf{x}^{t+1}, \mathbf{x}^{t}\right)+\left(2 L_{Y}-\sigma_{Y}\right) d_{h}\left(\mathbf{y}^{*}, \mathbf{y}^{t}\right)-2 L_{Y} d_{h}\left(\mathbf{y}^{*}, \mathbf{y}^{t+1}\right)$.

Summing up these two inequalities gives:

$$
\begin{aligned}
& f\left(\mathbf{x}^{t+1}, \mathbf{y}^{*}\right)-f\left(\mathbf{x}^{*}, \mathbf{y}^{t+1}\right) \\
& \quad \leq\left(2 L_{X}-\sigma_{X}\right) d_{g}\left(\mathbf{x}^{*}, \mathbf{x}^{t}\right)+\left(2 L_{Y}-\sigma_{Y}\right) d_{h}\left(\mathbf{y}^{*}, \mathbf{y}^{t}\right)-2 L_{X} d_{g}\left(\mathbf{x}^{*}, \mathbf{x}^{t+1}\right)-2 L_{Y} d_{h}\left(\mathbf{y}^{*}, \mathbf{y}^{t+1}\right) .
\end{aligned}
$$

Let $\sigma=\min \left\{\frac{\sigma_{X}}{L_{X}}, \frac{\sigma_{Y}}{L_{Y}}\right\}$. Then:

$$
\sum_{t=0}^{T-1}\left(\frac{2}{2-\sigma}\right)^{t}\left(f\left(\mathbf{x}^{t+1}, \mathbf{y}^{*}\right)-f\left(\mathbf{x}^{*}, \mathbf{y}^{t+1}\right)\right) \leq\left(2 L_{X}-\sigma_{X}\right) d_{g}\left(\mathbf{x}^{*}, \mathbf{x}^{0}\right)+\left(2 L_{Y}-\sigma_{Y}\right) d_{h}\left(\mathbf{y}^{*}, \mathbf{y}^{0}\right) .
$$

Note that $f\left(\mathbf{x}^{t}, \mathbf{y}^{*}\right)-f\left(\mathbf{x}^{*}, \mathbf{y}^{t}\right)$ is positive for each $t$, so the result follows.

\section{ANALYSIS OF DAMPED PROPORTIONAL RESPONSE}

Excluding Cobb-Douglas Utility Functions. First, we consider a simplified situation where there is no buyer with a Cobb-Douglas utility function. We want to use the technique developed in the saddle point analysis to obtain a convergence result. The potential function is the same as before.

We make the following observations.

Lemma 8.1. If $\rho_{i}>0$ for buyer $i$, then the Damped Proportional Response (given by (7)) is equivalent to mirror descent with a halved step size, defined as follows:

$$
\mathbf{b}_{i}^{t+1}=\arg \min _{\mathbf{b}_{i}: \sum_{j} b_{i j}=e_{i}}\left\{\left\langle\nabla_{\mathbf{b}_{i}} \Phi\left(\mathbf{b}^{t}\right), \mathbf{b}_{i}-\mathbf{b}_{i}^{t}\right\rangle+\frac{2}{\rho_{i}} \mathrm{KL}\left(\mathbf{b}_{i} \| \mathbf{b}_{i}^{t}\right)\right\} ;
$$

if $-\infty<\rho_{i}<0$ for buyer $i$, then the Damped Proportional Response (given by (7)) is equivalent to mirror descent (really ascent as this is a concave function) with a halved step size defined as follows:

$$
\mathbf{b}_{i}^{t+1}=\arg \min _{\mathbf{b}_{i}: \sum_{j} b_{i j}=e_{i}}\left\{-\left\langle\nabla_{\mathbf{b}_{i}} \Phi\left(\mathbf{b}^{t}\right), \mathbf{b}_{i}-\mathbf{b}_{i}^{t}\right\rangle+\frac{2\left(\rho_{i}-1\right)}{\rho_{i}} \mathrm{KL}\left(\mathbf{b}_{i} \| \mathbf{b}_{i}^{t}\right)\right\} ;
$$


and if $\rho_{i}=-\infty$ for buyer $i$, then the Damped Proportional Response (given by (7)) is equivalent to mirror descent (really ascent as this is a concave function) with a halved step sizem defined as follows:

$$
\mathbf{b}_{i}^{t+1}=\arg \min _{\mathbf{b}_{i}: \Sigma_{j} b_{i j}=e_{i}}\left\{-\left\langle\nabla_{\mathbf{b}_{i}} \Phi\left(\mathbf{b}^{t}\right), \mathbf{b}_{i}-\mathbf{b}_{i}^{t}\right\rangle+2 \mathrm{KL}\left(\mathbf{b}_{i} \| \mathbf{b}_{i}^{t}\right)\right\} .
$$

Proof. By calculation.

By Lemma 6.1 and with a simple calculation one can show that, in Definition 2, if we set $\mathbf{x}=\mathbf{b}_{>0}$, $\mathbf{y}=\mathbf{b}_{<0}, d_{g}(\mathbf{x})=\sum_{i: \rho_{i}>0} \frac{1}{\rho_{i}} \mathrm{KL}\left(\mathbf{b}_{i} \| \mathbf{b}_{i}^{\prime}\right)$, and $d_{h}(\mathbf{y})=\sum_{i: \infty<\rho_{i}<0} \frac{\rho_{i}-1}{\rho_{i}} \mathrm{KL}\left(\mathbf{b}_{i} \| \mathbf{b}_{i}^{\prime}\right)+\sum_{i: \rho_{i}=-\infty} \mathrm{KL}\left(\mathbf{b}_{i} \| \mathbf{b}_{i}^{\prime}\right)$ , then $\Phi$ is $(1,1)$-convex-concave function.

Furthermore, let $\mathbf{b}_{>0}^{*}$ and $\mathbf{b}_{<0}^{*}$ be the market equilibrium of the Fisher market. Then,

$$
\mathbf{b}_{>0}^{*} \text { minimizes } \Phi\left(\cdot, \mathbf{b}_{<0}^{*}\right) \text {, and } \mathbf{b}_{<0}^{*} \text { maximizes } \Phi\left(\mathbf{b}_{>0}^{*}, \cdot\right) \text {, }
$$

which implies $\left(\mathbf{b}_{>0}^{*}, \mathbf{b}_{<0}^{*}\right)$ is a saddle point of the potential function $\Phi$. Theorem 3.4 yields the following corollary.

COROLlary 8.2. The Damped Proportional Response (given by (7)) converges to the equilibrium with a convergence rate of:

$$
\begin{aligned}
& \sum_{t=1}^{T}\left[\Phi\left(\mathbf{b}_{>0}^{t}, \mathbf{b}_{<0}^{*}\right)-\Phi\left(\mathbf{b}_{>0}^{*}, \mathbf{b}_{<0}^{t}\right)\right] \\
& \leq \sum_{i:-\infty<\rho_{i}<0} \frac{2\left(\rho_{i}-1\right)}{\rho_{i}} \mathrm{KL}\left(\mathbf{b}_{i}^{*} \| \mathbf{b}_{i}^{0}\right)+\sum_{i: \rho_{i}>0} \frac{2}{\rho_{i}} \mathrm{KL}\left(\mathbf{b}_{i}^{*} \| \mathbf{b}_{i}^{0}\right)+\sum_{i: \rho_{i}=-\infty} 2 \mathrm{KL}\left(\mathbf{b}_{i}^{*} \| \mathbf{b}_{i}^{0}\right) .
\end{aligned}
$$

Moreover, if we assume there is no buyer with either a linear utility of a Leontief utility function, then, by Lemma $6.1, \Psi(\cdot, \cdot)$ is a $\left(\min _{i: \rho_{i}>0}\left\{1-\rho_{i}\right\}, \min _{i: \rho_{i}<0}\left\{\frac{1}{1-\rho_{i}}\right\}, 1,1\right)$-strong Bregman convex-concave function with $\mathbf{x}=\mathbf{b}_{>0}, \mathbf{y}=\mathbf{b}_{<0}, d_{g}(\mathbf{x})=\sum_{i: \rho_{i}>0} \frac{1}{\rho_{i}} \mathrm{KL}\left(\mathbf{b}_{i} \| \mathbf{b}_{i}^{\prime}\right)$ and $d_{h}(\mathbf{y})=$ $\sum_{i: \infty<\rho_{i}<0} \frac{\rho_{i}-1}{\rho_{i}} \mathrm{KL}\left(\mathbf{b}_{i} \| \mathbf{b}_{i}^{\prime}\right)$ (see Definition 4). Theorem 3.5 yields the following corollary.

COROLLARY 8.3. Suppose there is no buyer with either a linear utility or a Leontief utility. Let

$$
\sigma_{>0}=\min _{i: \rho_{i}>0}\left\{1-\rho_{i}\right\} \text { and } \sigma_{<0}=\min _{i: \rho_{i}<0}\left\{\frac{1}{1-\rho_{i}}\right\} \text {. }
$$

Then the Damped Proportional Response (given by (7)) converges to the equilibrium with a convergence rate of

$$
\begin{aligned}
& \Phi\left(\mathbf{b}_{>0}^{T}, \mathbf{b}_{<0}^{*}\right)-\Phi\left(\mathbf{b}_{>0}^{*}, \mathbf{b}_{<0}^{T}\right) \\
& \quad \leq\left(1-\frac{\min \left\{\sigma_{>0}, \sigma_{<0}\right\}}{2}\right)^{T-1}\left[\sum_{i: \rho_{i}<0}\left(2-\sigma_{<0}\right) \frac{\rho_{i}-1}{\rho_{i}} \mathrm{KL}\left(\mathbf{b}_{i}^{*} \| \mathbf{b}_{i}^{0}\right)+\sum_{i: \rho_{i}>0}\left(2-\sigma_{>0}\right) \frac{1}{\rho_{i}} \mathrm{KL}\left(\mathbf{b}_{i}^{*} \| \mathbf{b}_{i}^{0}\right)\right] .
\end{aligned}
$$

The Entire CES Range Now we consider the Damped Proportional Response with a damping factor of 2 over the entire CES range, i.e. including Cobb-Douglas utilities. Recall that we modify our potential function to include terms for the buyers with $\rho_{i}=0$. However, for this new modified function, the buyers with Cobb-Douglas utility functions don't actually perform mirror descent. Fortunately, we can make two observations.

First, the buyers with Cobb-Douglas utility functions converge quickly to the equilibrium independently of everyone else's spending. Second, the buyers whose utility functions are not Cobb-Douglas will still perform the mirror descent (ascent) procedure. 
So, intuitively, in our analysis, we regard the spending of the buyers with Cobb-Douglas utility functions as a parameter, $\theta$, of $f_{\theta}(\mathbf{x}, \mathbf{y})$, where $\mathbf{x}$ represents the spending of the strictly substitutes buyers and $y$ represents the spending of the strictly complementary buyers. Remember, in the case with no Cobb-Douglas utilities, the market equilibrium corresponded to a saddle point. Here, similarly, a market equilibrium corresponds to a saddle point of $f_{\theta^{*}}(\cdot, \cdot)$, where $\theta^{*}$ is the spending at the market equilibrium of those buyers with Cobb-Douglas utility functions. We prove the following two claims.

(1) $\theta$ converges to $\theta^{*}$ quickly;

(2) when $\theta$ tends to $\theta^{*}$, though $\mathbf{x}$ and $\mathbf{y}$ perform the mirror descent based on the gradient of $f_{\theta}(\mathbf{x}, \mathbf{y})$ and not of $f_{\theta^{*}}(\mathbf{x}, \mathbf{y}),(\mathbf{x}, \mathbf{y})$ will still converge quickly to $\left(\mathbf{x}^{*}, \mathbf{y}^{*}\right)$, the saddle point of $f_{\theta^{*}}(\cdot, \cdot)$.

We thereby show that Damped Proportional Response converges to the market equilibrium even when faced with the entire range of CES utilities.

\section{OTHER MEASURES OF CONVERGENCE}

The potential function $\phi$ appears to be closely related to the Eisenberg-Gale program. In particular, we can show that in the substitutes domain, when applying update rule (2), the Proportional Response update, the objective function $\Psi$ for the Eisenberg-Gale program converges at least as fast as $\Phi$, i.e. that $\Psi\left(\mathbf{x}\left(\mathbf{b}_{>0}^{*}, \mathbf{b}_{=0}^{*}\right)\right)-\Psi\left(\mathbf{x}\left(\mathbf{b}_{>0}, \mathbf{b}_{=0}^{*}\right)\right) \leq \Phi\left(\mathbf{b}_{>0}, \mathbf{b}_{=0}^{*}\right)-\Phi\left(\mathbf{b}_{>0}^{*}, \mathbf{b}_{=0}^{*}\right)$, and that in the complementary domain, when applying update rule (3), the objective function for the dual of the Eisenberg-Gale program converges at least as fast as $\Phi$. These claims are shown in the full paper.

Lemma 6.1 allows us to make some observations about the rate of convergence of the spending. For update rule (2), in the substitutes domain excluding linear utilities, we can deduce that $\sum_{i} \mathrm{KL}\left(\mathbf{b}_{i} \| \mathbf{b}_{i}^{*}\right) \leq \max _{i} \frac{\rho_{i}}{1-\rho_{i}}\left[\Phi(\mathbf{b})-\Phi\left(\mathbf{b}^{*}\right)\right]$, and for update rule (3) in the complementary domain excluding Leontief utilities, that $\sum_{i} \mathrm{KL}\left(\mathbf{b}_{i} \| \mathbf{b}_{i}^{*}\right) \leq \max _{i}-\rho_{i}\left[\Phi(\mathbf{b})-\Phi\left(\mathbf{b}^{*}\right)\right]$. As the equilibrium need not be unique in terms of spending for either linear or Leontief utilities, this lemma is not going to yield a bound on the convergence rate of the spending in these cases, as it can be applied to any equilibrium. Similarly in the combined domain, still excluding linear and Leontief utilities, we can observe that

$$
\sum_{i: \rho_{i}>0} \frac{1-\rho_{i}}{\rho_{i}} \mathrm{KL}\left(\mathbf{b}_{i} \| \mathbf{b}_{i}^{*}\right)+\sum_{i: \rho_{i}<0} \frac{-1}{\rho_{i}} \mathrm{KL}\left(\mathbf{b}_{i} \| \mathbf{b}_{i}^{*}\right) \leq\left[\Phi\left(\mathbf{b}_{>0}, \mathbf{b}_{<0}^{*}\right)-\Phi\left(\mathbf{b}^{*}\right)\right]+\left[\Phi\left(\mathbf{b}^{*}\right)-\Phi\left(\mathbf{b}_{>0}^{*}, \mathbf{b}_{<0}\right)\right] .
$$

In addition, since $\mathrm{KL}\left(\mathbf{p} \| \mathbf{p}^{*}\right) \leq \mathrm{KL}\left(\mathbf{b} \| \mathbf{b}^{*}\right)$ we can immediately obtain analogous bounds on the KL divergence of the prices. Furthermore, for the substitutes domain, including linear utilities, Lemma 6.1 also implies that $\mathrm{KL}\left(\mathbf{p} \| \mathbf{p}^{*}\right) \leq\left[\Phi(\mathbf{b})-\Phi\left(\mathbf{b}^{*}\right)\right]$.

\section{ACKNOWLEDGMENTS}

We are grateful to the anonymous referees for their insightful comments which helped improve the presentation.

\section{REFERENCES}

[1] Kenneth J. Arrow, H. D. Block, and Leonid Hurwicz. 1959. On the Stability of Competitive Equilibrium, II. Econometrica 27, 1 (1959), 82-109. 
[2] Benjamin Birnbaum, Nikhil R. Devanur, and Lin Xiao. 2011. Distributed Algorithms via Gradient Descent for Fisher Markets. In Proceedings of the 12th ACM Conference on Electronic Commerce (EC '11). ACM, 127-136. DOI: http: //dx.doi.org/10.1145/1993574.1993594

[3] Lawrence E. Blume. 1993. The Statistical Mechanics of Strategic Interaction. Games and Economic Behavior 5, 3 (1993), 387-424.

[4] Eric Budish. 2011. The Combinatorial Assignment Problem: Approximate Competitive Equilibrium from Equal Incomes. Journal of Political Economy 119, 6 (2011), 1061-1103.

[5] Ioannis Caragiannis, David Kurokawa, Hervé Moulin, Ariel D. Procaccia, Nisarg Shah, and Junxing Wang. 2016. The Unreasonable Fairness of Maximum Nash Welfare. In Proceedings of the 2016 ACM Conference on Economics and Computation (EC '16). ACM, 305-322. DOI : http://dx.doi.org/10.1145/2940716.2940726

[6] Gong Chen and Marc Teboulle. 1993. Convergence Analysis of a Proximal-Like Minimization Algorithm Using Bregman Functions. SIAM fournal on Optimization 3, 3 (1993), 538-543.

[7] X. Chen, D. Dai, Y. Du, and S. H. Teng. 2009. Settling the Complexity of Arrow-Debreu Equilibria in Markets with Additively Separable Utilities. In 2009 50th Annual IEEE Symposium on Foundations of Computer Science. 273-282. DOI : http://dx.doi.org/10.1109/FOCS.2009.29

[8] Xi Chen, Xiaotie Deng, and Shang-Hua Teng. 2009. Settling the Complexity of Computing Two-player Nash Equilibria. f. ACM 56, 3, Article 14 (May 2009), 57 pages.

[9] Xi Chen, Dimitris Paparas, and Mihalis Yannakakis. 2017. The Complexity of Non-Monotone Markets. J. ACM 64, 3 (2017), 20:1-20:56. DOI : http://dx.doi.org/10.1145/3064810

[10] Xi Chen and Shang-Hua Teng. 2009. Spending Is Not Easier Than Trading: On the Computational Equivalence of Fisher and Arrow-Debreu Equilibria. In Algorithms and Computation, 20th International Symposium, ISAAC 2009, Honolulu, Hawaii, USA, December 16-18, 2009. Proceedings. 647-656. DOI : http://dx.doi.org/10.1007/978-3-642-10631-6_66

[11] Yun Kuen Cheung, Richard Cole, and Nikhil Devanur. 2013. Tatonnement Beyond Gross Substitutes?: Gradient Descent to the Rescue. In Proceedings of the Forty-fifth Annual ACM Symposium on Theory of Computing (STOC '13). ACM, 191-200. DOI : http://dx.doi.org/10.1145/2488608.2488633

[12] Yun Kuen Cheung, Richard Cole, and Ashish Rastogi. 2012. Tatonnement in Ongoing Markets of Complementary Goods. In Proceedings of the 13th ACM Conference on Electronic Commerce (EC '12). ACM, 337-354. D0I: http://dx.doi. org/10.1145/2229012.2229039

[13] Bruno Codenotti, Benton McCune, and Kasturi Varadarajan. 2005. Market Equilibrium via the Excess Demand Function. In Proceedings of the Thirty-seventh Annual ACM Symposium on Theory of Computing (STOC '05). ACM, 74-83. http://doi.acm.org/10.1145/1060590.1060601

[14] Bruno Codenotti, Amin Saberi, Kasturi Varadarajan, and Yinyu Ye. 2006. Leontief Economies Encode Nonzero Sum Two-player Games. In Proceedings of the Seventeenth Annual ACM-SIAM Symposium on Discrete Algorithm (SODA '06). Society for Industrial and Applied Mathematics, 659-667.

[15] Richard Cole, Nikhil Devanur, Vasilis Gkatzelis, Kamal Jain, Tung Mai, Vijay V. Vazirani, and Sadra Yazdanbod. 2017. Convex Program Duality, Fisher Markets, and Nash Social Welfare. In Proceedings of the 2017 ACM Conference on Economics and Computation (EC '17). ACM, 459-460. DOI : http://dx.doi.org/10.1145/3033274.3085109

[16] Richard Cole and Lisa Fleischer. 2008. Fast-converging Tatonnement Algorithms for One-time and Ongoing Market Problems. In Proceedings of the Fortieth Annual ACM Symposium on Theory of Computing (STOC '08). ACM, 315-324. DOI : http://dx.doi.org/10.1145/1374376.1374422

[17] Constantinos Daskalakis, Paul W. Goldberg, and Christos H. Papadimitriou. 2009. The Complexity of Computing a Nash Equilibrium. SIAM J. Comput. 39, 1 (2009), 195-259.

[18] Xiaotie Deng and Ye Du. 2008. The Computation of Approximate Competitive Equilibrium is PPAD-hard. Inf. Process. Lett. 108, 6 (Nov. 2008), 369-373.

[19] Xiaotie Deng, Christos Papadimitriou, and Shmuel Safra. 2003. On the complexity of price equilibria. Fournal of Computer System Sciences (FCSS) 67 (2003), 311-324.

[20] Nikhil R. Devanur. 2004. The Spending Constraint Model for Market Equilibrium: Algorithmic, Existence and Uniqueness Results. In Proceedings of the Thirty-sixth Annual ACM Symposium on Theory of Computing (STOC '04). ACM, 519-528. DOI : http://dx.doi.org/10.1145/1007352.1007431

[21] Nikhil R. Devanur, Christos H. Papadimitriou, Amin Saberi, and Vijay V. Vazirani. 2008. Market equilibrium via a primal-dual algorithm for a convex program. f. ACM 55, 5 (2008), 22:1-22:18.

[22] Krishnamurthy Dvijotham, Yuval Rabani, and Leonard J. Schulman. 2017. Convergence of Incentive-driven Dynamics in Fisher Markets. In Proceedings of the Twenty-Eighth Annual ACM-SIAM Symposium on Discrete Algorithms (SODA '17). Society for Industrial and Applied Mathematics, 554-567.

[23] E. Eisenberg. 1961. Aggregation of Utility Functions. Management Sciences 7 (1961), 337-350.

[24] E. Eisenberg and D. Gale. 1959. Consensus of subjective probabilities: the Pari-Mutuel method. The Annals of Mathematical Statistics 30 (1959), 165-168. 
[25] Rahul Garg and Sanjiv Kapoor. 2006. Auction Algorithms for Market Equilibrium. Math. Oper. Res. 31, 4 (Nov. 2006), 714-729.

[26] Gauthier Gidel, Tony Jebara, and Simon Lacoste-Julien. 2017. Frank-Wolfe Algorithms for Saddle Point Problems. In The 20th International Conference on Artificial Intelligence and Statistics.

[27] Aanund Hylland and Richard Zeckhauser. 1979. The Efficient Allocation of Individuals to Positions. Journal of Political Economy 87, 2 (April 1979), 293-314.

[28] Kamal Jain and Vijay V. Vazirani. 2007. Eisenberg-Gale Markets: Algorithms and Structural Properties. In Proceedings of the Thirty-ninth Annual ACM Symposium on Theory of Computing (STOC '07). ACM, 364-373. DOI : http://dx.doi.org/ 10.1145/1250790.1250845

[29] Mamoru Kaneko and Kenjiro Nakamura. 1979. The Nash social welfare function. Econometrica (1979), 423-435.

[30] Frank Kelly. 1997. Charging and rate control for elastic traffic. European Transactions on Telecommunications 8, 1 (1997), 33-37.

[31] Dave Levin, Katrina LaCurts, Neil Spring, and Bobby Bhattacharjee. 2008. Bittorrent is an Auction: Analyzing and Improving Bittorrent's Incentives. SIGCOMM Comput. Commun. Rev. 38, 4 (Aug. 2008), 243-254. DOI : http: //dx.doi.org/10.1145/1402946.1402987

[32] Steven H Low and David E Lapsley. 1999. Optimization flow control. I. Basic algorithm and convergence. IEEE/ACM Transactions on networking 7, 6 (1999), 861-874.

[33] Jason R. Marden and Jeff S. Shamma. 2012. Revisiting log-linear learning: Asynchrony, completeness and payoff-based implementation. Games and Economic Behavior 75, 2 (2012), 788-808.

[34] L. W. McKenzie. 2002. Classical General Equilibrium Theory. The MIT press.

[35] J. F. Nash. 1950. The Bargaining Problem. Econometrica 18 (1950), 155-162.

[36] Arkadi Nemirovski. 2004. Prox-Method with Rate of Convergence O(1/t) for Variational Inequalities with Lipschitz Continuous Monotone Operators and Smooth Convex-Concave Saddle Point Problems. SIAM fournal on Optimization 15,1 (2004), 229-251.

[37] Yurii Nesterov. 2005. Excessive Gap Technique in Nonsmooth Convex Minimization. SIAM fournal on Optimization 16, 1 (2005), 235-249.

[38] Yurii Nesterov. 2005. Smooth minimization of non-smooth functions. Math. Program. 103, 1 (2005), 127-152.

[39] Yurii Nesterov. 2007. Dual extrapolation and its applications to solving variational inequalities and related problems. Mathematical Programming 109, 2-3 (2007), 319-344.

[40] Noam Nisan, Tim Roughgarden, Eva Tardos, and Vijay V. Vazirani. 2007. Algorithmic Game Theory. Cambridge University Press, New York, NY, USA.

[41] Christos H. Papadimitriou and Mihalis Yannakakis. 2010. An Impossibility Theorem for Price-adjustment Mechanisms. PNAS 5, 107 (2010), 1854-1859.

[42] Alexander Rakhlin and Karthik Sridharan. 2013. Optimization, Learning, and Games with Predictable Sequences. In Advances in Neural Information Processing Systems 26: Twenty-seventh Annual Conference on Neural Information Processing Systems 2013. 3066-3074.

[43] H. Scarf. 1960. Some examples of global instatibility of the competitive equilibrium. International Economic Review 3 , 13 (1960), 157-172.

[44] Vadim I Shmyrev. 2009. An algorithm for finding equilibrium in the linear exchange model with fixed budgets. fournal of Applied and Industrial Mathematics 3, 4 (2009), 505.

[45] Hirofumi Uzawa. 1960. Walras' Tatonnement in the Theory of Exchange. Review of Economic Studies 27, 3 (1960), 182-194.

[46] H.R. Varian. 1974. Equity, envy, and efficiency. Journal of Economic Theory 9, 1 (1974), 63-91.

[47] Vijay V. Vazirani and Mihalis Yannakakis. 2011. Market equilibrium under separable, piecewise-linear, concave utilities. 7. ACM 58, 3 (2011), 10:1-10:25.

[48] Léon Walras. 1874. Eléments d'Economie Politique Pure. Corbaz. (Translated as: Elements of Pure Economics. Homewood, IL: Irwin, 1954.).

[49] Fang Wu and Li Zhang. 2007. Proportional Response Dynamics Leads to Market Equilibrium. In Proceedings of the Thirty-ninth Annual ACM Symposium on Theory of Computing (STOC '07). ACM, 354-363. DOI : http://dx.doi.org/10. 1145/1250790.1250844

[50] Li Zhang. 2011. Proportional response dynamics in the Fisher market. Theor. Comput. Sci. 412, 24 (2011), $2691-2698$. 